D.Y. Aksin, F. Maloberti: "Non-Linear Behavioral Model of a Bipolar Trackand-Hold Amplifier for High-Speed and High-Resolution ADCs"; 12th IEEE Int. Conf. on Electronics, Circuits and Systems, ICECS 2005, Gammarth, 11-14 December 2005.

(C)20xx IEEE. Personal use of this material is permitted. However, permission to reprint/republish this material for advertising or promotional purposes or for creating new collective works for resale or redistribution to servers or lists, or to reuse any copyrighted component of this work in other works must be obtained from the IEEE. 


\title{
Non-Linear Behavioral Model of a Bipolar Track and Hold Amplifier For High-Speed and High-Resolution ADCs Devrim Y. Aksin ${ }^{1,3}$ and Franco Maloberti ${ }^{2}$
}

\author{
${ }^{1}$ Department of Electrical Engineering University of Texas at Dallas, Richardson, TX, USA \\ ${ }^{2}$ Department of Electrical Engineering University of Pavia, Pavia, Italy \\ ${ }^{3}$ Mixed Signal Power and Control Group, Texas Instruments Inc, Dallas, TX, USA
}

\begin{abstract}
An effective non-linear behavioral model of a bipolar track-and-hold amplifier (THA) is described. The model is aimed for system level simulations and provides accurate and fast simulation. The model is written with AHDL. Basic THA properties together with noise and non-linearity features are modeled with a simulation time improvement, for high accuracy setup, up to 7 times of the transistor level. Extensive simulations validate the model and prove the benefits.
\end{abstract}

KeyWords: System level Behavioral modeling, Bipolar Track-and-hold Amplifier, High-speed, Data conversion.

\section{Introduction}

A track-and-hold amplifier (THA) is the front-end of any high-end Analog-to-Digital Converter (ADC). The input is tracked during one phase and kept for the entire hold period. The output is suitable for the successive quantization if the accuracy of the THA is good enough for achieving required resolution. If the THA is used for IF-sampling within RF receivers, the harmonic degradation is key for obtaining a proper I/Q separation at the DSP level [1].

The ADC (including the THA) is normally part of an electronic system. The simulations at the system level determine the limits of critical blocks and quantify specifications of sub-blocks. The complexity of the system is often such that the simulations at transistor level require extremely long CPU times. Thus, it is necessary to use accurate high-level behavioral models to speed up system level design process.

This paper describes behavioral model of a bipolar THA, which: 1) uses behavioral parameters that are derived from a set of transistor-level simulations, 2) describes the THA features at different level of complexity, 3) determines system level parameters, like spurious free dynamic range, signal-to-noise ratio, slew rate, power, before designing sub-blocks at a fraction of the time required by the transistor level counterpart.

The AHDL behavioral model accounts for basic THA properties such as: input loading, tracking bandwidth, output clipping, output current limit and output resistance. Moreover, several dynamic non-idealities such as clock jitter induced noise, kT/C noise, supply noise, harmonic distortion, and clock feed-through, are also included.

\section{Model Description}

The block diagram of Fig. 1 shows the relations among various blocks describing linear and non-linear behavior of the THA given in Fig. 2. This is a push-pull emitter follower made by the cascade of two emitter followers, one with npn and the other with pnp input [2]. During the track mode, the switches convert the current sources and the transistors $\mathrm{Q}_{1}$ and $\mathrm{Q}_{2}$ to two emitter followers. The push pull transistors transfer the signal to the output. The function of each block in Fig.1 is written in AHDL.

The input voltage is applied to an input stage that performs a linear preprocessing accounting for the input impedance. The output of this block drives the blocks that model four dynamic non-idealities: the charge injection and the hold-mode droop (these are modeled by the same block), tracking mode harmonic distortion and the hold mode feed-through. The output of the input stage together with the offset and the noise is applied to a low-pass linear filter to model tracking bandwidth. The harmonic distortion signals are then added to create the signal at the end of the sampling phase. The THA core incorporates the charge injection and the hold mode droop to have an intermediate output signal that is later summed with supply noise and hold mode feedthrough to drive output stage. The last block accounts for the distortion and the finite driving capability of the output stage.

\section{Behavioral Blocks}

The input stage is simply a parallel RC network modeling the input load of the THA. Then, after a buffer, DC offset and noise is added to the input. The offset 
consists of an input parameter plus $1^{\text {st }}$ order temperature drift term. The noise generators of the active THA determine a global noise contribution across the sampling capacitor $\mathrm{C}_{\mathrm{HOLD}}$. The global effect is referred to the input and is proportional to the well-known $\mathrm{kT} / \mathrm{C}$ noise power. The noise generator model is

$$
V_{T H}=\sqrt{\frac{k T}{C_{H O L D}}} \delta_{t h}
$$

where $\delta_{\text {th }}$ is a random number whose variance is a parameter of the input deck. The noise generator also includes the effect of clock jitter. The uncertainty on sampling instant is modeled with an additional noise generator given by

$$
V_{\text {JITTER }}\left(t_{S}\right)=\left.\delta_{j i} \frac{d}{d t} V_{I N}(t)\right|_{t=t_{S}}
$$

where $\delta_{\mathrm{ji}}$ is a random number whose variance is another parameter of the input deck [3]. The main problem of implementing (1) and (2), is the dependency of the output noise power on the simulation time step due to the interpolation error introduced during FFT [4]. As oppose to the classical approach, our model creates the noise pattern prior to simulation to ensure the consistency of the extracted dynamic performance and to speed up the simulation. The noise power is only important for the simulations used to extract the sampled system's dynamic range. These simulations generally run for predetermined number of cycles that is fixed by FFT. Our behavioral model uses this information to determine the noise signal before hand to make the noise power independent of the simulation time step and consistent throughout multiple simulations. This dramatically improves the simulation speed. The signal at the output of the low-pass filter is

$$
V_{\text {FILTER }}=\frac{1}{1+\frac{s}{2 \pi f_{T B W}}}\left(V_{I N}+V_{o f f s e t}+V_{\text {JITTER }}+V_{T H}\right)
$$

The model used for accounting harmonic distortion uses two coefficients, i.e. $a_{2}$ and $a_{3}$

$$
V_{H A R}=a_{2} V_{I N}^{2}+a_{3} V_{I N}^{3}
$$

these coefficients are non-linear and depend on the input amplitude and frequency. The distortion coefficients are calculated using the nonlinearity analysis given in [5]. The set of equations used depends on the specific THA topology, its bias and the technology. Therefore, for any topology, it is necessary to develop a specific model that provides equations used by the behavioral description for estimating the distortion coefficients. The complexity of the models used in the nonlinear analysis depends on the number of non-linear devices accounted for. The designer identifies the critical active devices and decides the ones that are worth including in the non-linear study.

For the THA shown in Fig. 2, a high frequency input signal causes a significant current flowing into the sampling capacitor. Thus, the main high-frequency distortion contributors during the track mode are $Q_{3}$ and $\mathrm{Q}_{4}$. The effect of transistors $\mathrm{Q}_{1}$ and $\mathrm{Q}_{2}$ is more limited since we have only parasitic terms at the emitter nodes. Finally the contribution of transistors making the switches is negligible. Similar discussions apply for the hold mode. Hence, the nonlinear analysis includes only the non-linearity of $Q_{3}$ and $Q_{4}$.

Different levels of coefficient accuracy are obtained using device models with different accuracy. If the model is very accurate the equations become complex yielding longer simulation time. Our code uses two levels of device model accuracy. Simulations show that the difference in results is negligible at high frequency. At low frequency the clock feed-through non-linearity is the dominant term [6]. Thus, the complex model is necessary only for measuring the track-mode nonlinearity.

The functional operation of the THA Core is shown in Fig. 3. During the hold mode, the charge injection and the hold mode droop are the two limits that modify the held voltage. During this phase, the multiplying factor is switched to one and the voltage source shifts the $\mathrm{C}_{\mathrm{HOLD}}$ bottom plate according to the calculated charge injection and hold mode droop. Proposed technique improves the accuracy and the convergence of the simulator.

The model of the voltage shift accounting for charge injection depends on the value of the input voltage, the bias current and the waveform of the switching clock. The transition from track to hold with zero input gives a fixed offset. A given input leads to a non-linear change of the equivalent offset. Obviously, the offset is inversely proportional to the hold capacitance. The model stores the information for a given THA architecture using polynomial descriptions of the equivalent offset. A relatively small number of DC simulations and best curve fitting calculations are enough to extract polynomial coefficients.

The output block controls the swing of the output voltage, limits the output current and models the output resistance. The soft clipping of output voltage is obtained using a voltage dependent gain. The gain reduction is a sigmoid function that mimics the 
saturation to the supply rails. This technique facilitates numerical convergence. A feed-forward current correction technique that monitors the output current through output resistance, bounds the output current. An additional block models the output load current and static supply current.

The code adaptively changes the maximum time step with clock, input signal frequency and the circuit's operation mode, i.e. track and hold mode as parameters. Required time resolution during the hold mode is less stringent than it is in the track mode. Accordingly, the time-step controller relaxes the maximum time step after switching to hold. This permits to reduce the simulation time by half while preserving the accuracy.

Transistor level DC simulations determine charge injection related parameters used in the behavioral code. The parameters are extracted using a "parameter acquisition" engine. It exercises the THA with suitable stimulus and obtains and stores in a file the behavioral parameters. Therefore, simulation flow is: 1) design the cell at the transistor level, 2) run the acquisition parameter routine, 3 ) use the behavioral code with the extracted parameter files.

\section{Simulation and Model Verification}

The behavioral model and the THA of Fig. 2 have been used to verify accuracy and simulation time benefits.

Fig. 4 shows a sampled sine wave and its power spectrum is given in Fig. 5. Simulation conditions are $\mathrm{V}_{\mathrm{IN}} 2 \mathrm{~V}_{\mathrm{PP}}$, input frequency $15.2 \mathrm{MHz}$, sampling frequency $200 \mathrm{MHz}, \mathrm{V}_{\mathrm{DD}} 5 \mathrm{~V}$, rms jitter $1 \mathrm{ps}$, bias current $1 \mathrm{~mA}, \mathrm{C}_{\text {Hold }} 1 \mathrm{pF}$, and temperature $27 \mathrm{C}$. The SNR and SFDR and SINAD are extracted from 4096 points FFT. Table 1 compares different parameters extracted from the circuit level THA and the code. Results' accuracy is quite satisfactory. Simulation results reveal that proposed technique models the charge injection with less than $2 \%$ error. Fig. 6 compares the harmonic distortion response of the transistor level THA and the behavioral code. The results are shifted with respect to one another by $2-3 \mathrm{~dB}$ with the behavioral results more pessimistic than the transistor level results. In both cases the harmonics jump up above $10 \mathrm{MHz}$.

The accuracy of the behavioral model enables interesting observations like the one shown in Fig. 7. As expected, for low values the SNR improves with increasing $\mathrm{C}_{\mathrm{HOLD}}$; the clock feed-through and charge
Table 1. Static Parameter comparison

\begin{tabular}{|l|c|c|c|c|}
\hline Parameter & Trans. & Code & Unit & Error \\
\hline Gain & $-4.5 \mathrm{~m}$ & $-4.5 \mathrm{~m}$ & {$[\mathrm{~dB}]$} & - \\
\hline Bandwidth & 8.33 & 8.27 & {$[\mathrm{GHz}]$} & $0.7 \%$ \\
\hline HMFF $@ 1 \mathrm{~Hz}$ & -240 & -240 & {$[\mathrm{~dB}]$} & - \\
\hline $\mathrm{R}_{\text {OUT }}$ & 19.17 & 19.17 & {$[\Omega]$} & - \\
\hline $\mathrm{V}_{\text {CLIP }}$ & 1 & 0.991 & {$[\mathrm{~V}]$} & $0.9 \%$ \\
\hline $\mathrm{I}_{\text {SUPPL }}$ & 3 & 3 & {$[\mathrm{~mA}]$} & - \\
\hline
\end{tabular}

Table 2. Simulation Time Comparison

\begin{tabular}{|l|c|c|c|}
\hline Tolerances $^{1}$ & Trans. & Code & Ratio \\
\hline Very Tight & $3069 \mathrm{~s}$ & $435 \mathrm{~s}$ & 7 \\
\hline Tight & $579 \mathrm{~s}$ & $175 \mathrm{~s}$ & 3.3 \\
\hline Relaxed & $19.1 \mathrm{~s}$ & $13 \mathrm{~s}$ & 1.5 \\
\hline
\end{tabular}

injection determines the SFDR for low $\mathrm{C}_{\mathrm{HOLD}}$ values. However, increasing the capacitance augments the capacitive currents and the track mode nonlinearities become dominant. As a result there is an optimum hold capacitance value for the SFDR.

Fig. 8 shows the process load caused by different subblocks of the code. THA core requires only $2 \%$ of total simulation time. The 5\% supply model includes supply current model and supply noise. Distortion Equation 1 and 2 correspond to a simple and a complex tracking distortion model. Most of the model blocks require a limited and comparable computation time. Instead the time required for the charge injection calculation is $24 \%$. More time is required for the track mode distortion: it is $31 \%$ with a simplified model and an additional $23 \%$ for a refined model.

Table 2 compares the transient simulation times of transistor level circuit with AHDL code for three different accuracy settings ${ }^{(1)}$. The benefit is a trade-off between accuracy (which is very good for this model) and simulation time. With relaxed tolerances, the results become increasingly approximate and the simulation time for the transistor level is comparable with the time required by the code. As a result the behavioral model is convenient only for accurate system level simulations.

\section{References}

[1] B. Razavi, "Next-generation RF circuits and systems", in Proc. $17^{\text {th }}$ Conf. Advanced Research in VLSI, 1997, pp.270-282.

[2] C. Moreland, M. Elliott, F. Murden, J. Young, M. Hensley and R. Stop, 'A 14-bit 100MSample/s subranging ADC', IEEE Journal of Solid-State Circuit, vol. 35, pp. 1791-1798, Dec. 2000.

\footnotetext{
${ }^{1}$ abstol, reltol and maximum transient step.
} 
[3] S. R. Norsworthy, R. Schreier, and G. C. Temes, "Delta-sigma data converters, in Theory, Design and Simulation", IEEE Press Piscataway, NJ, 1997.

[4] Private conversation with Bob Atwell.

[5] Piet Wambacq and Willy Sansen, "Distortion Analysis of Analog Integrated Circuits", Kluwer Academic Pub., Dordrecht, The Netherlands, 1998.

[6] Aksin, D.Y.; Maloberti, F. "Very high-speed BJT buffer for track-and-hold amplifiers with enhanced linearity", ISCAS 2002, vol. 5, pp. 569-572, May 2002.

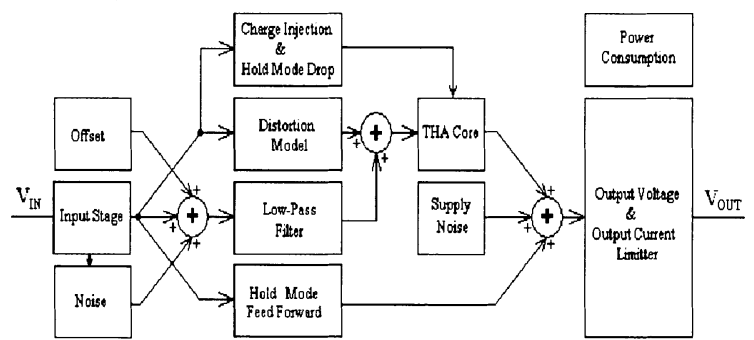

Figure 1. Functional Block Diagram of THA Model

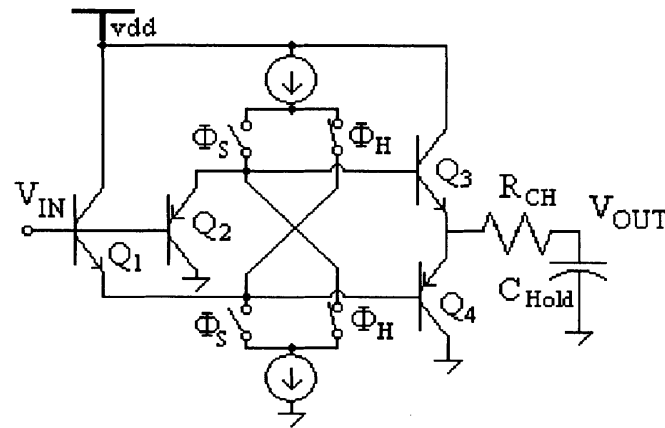

Figure 2. Modeled Bipolar Track and Hold Amplifier

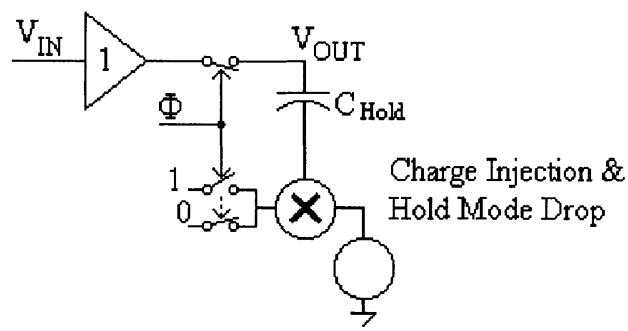

Figure 3. Track and Hold Amplifier Core.

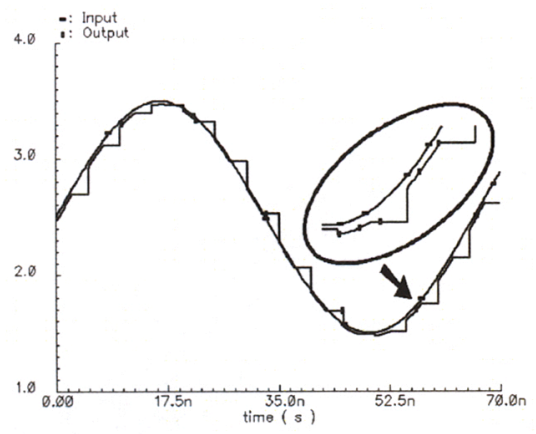

Figure 4. Sampled Sine Wave

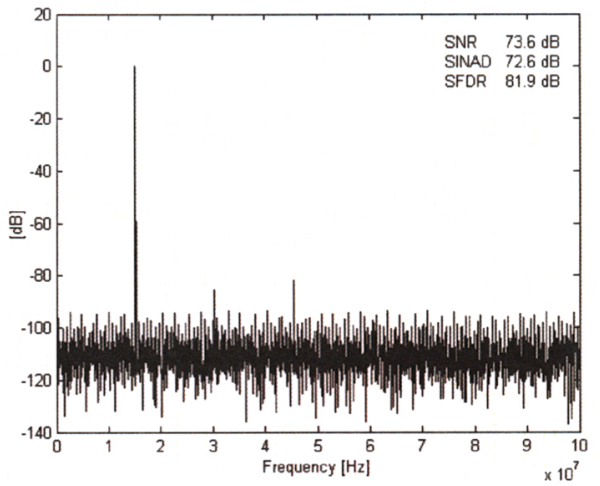

Figure 5. Sampled Sine Wave power spectrum

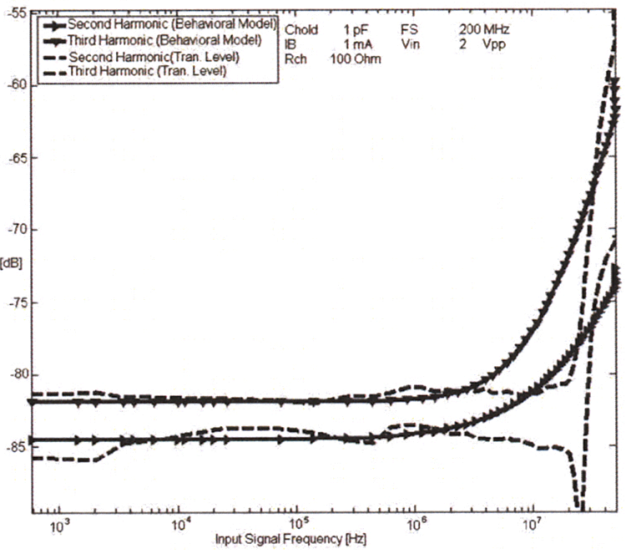

Figure 6. Harmonic distortion comparison.

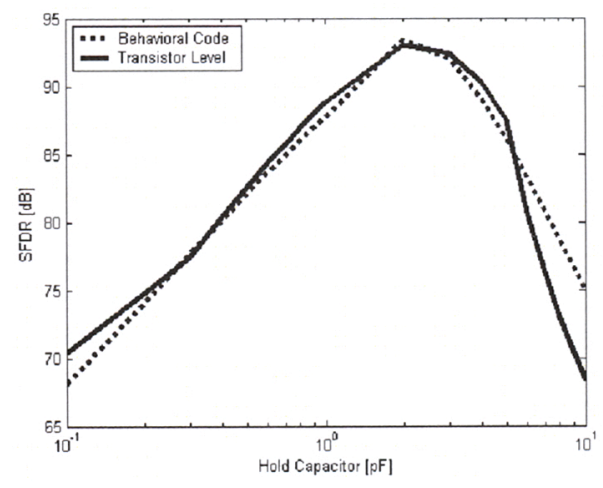

Figure 7. SFDR versus the hold capacitance.

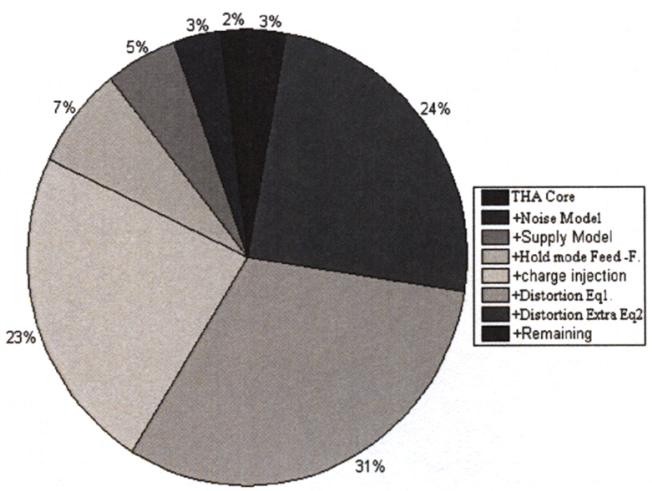

Figure 8. Process Load distribution. 